\title{
Critical points of the clamped-pinned elastica
}

Received: 12 October 2017 / Revised: 4 May 2018 / Published online: 15 September 2018 (C) The Author(s) 2018

\begin{abstract}
We investigate equilibrium configurations of the clamped-pinned elastica where the pinned end can be displaced towards, and past, the clamped end. Solving the nonlinear ordinary differential equation for the clamped-pinned elastica for any mode in terms of elliptic integrals, we find sets of equations which govern the equilibrium configurations for given displacements. Equilibrium configurations for various displacements of the pinned end and any mode are obtained by numerically solving those sets of equations. A physical quantity, such as the force that arises in the elastica due to displacement of the pinned end, is taken to be a function of displacement. Although it is generally not possible to define a physical quantity as a function of displacement explicitly, an equation for the rate of change of this physical quantity with respect to displacement can be found by partial differentiation of the sets of equations which govern the equilibrium configurations. Setting that rate of change to zero provides a constraint equation for locating the critical points of that physical quantity. That constraint equation and the sets of equations which govern the equilibrium configurations are solved numerically to obtain the critical points of the physical quantity. Our procedure is demonstrated by finding local extrema on force-displacement plots (useful when analysing the stability of equilibrium configurations) and the maximum deflection of the elastica. Finally, we suggest how our procedure has scope for wider application.
\end{abstract}

\section{Introduction}

In elasticity theory, the problem of the elastica finds applications in many fields of mechanics and increasingly biomechanics [1-3]. The equilibrium solutions of the elastica depend on the intrinsic state of the rod, the constitutive relations, the loading conditions, and boundary conditions. Though the elastica is a nonlinear problem, some cases can be solved exactly by elliptic functions and integrals. There may also be multiple solutions for a given loading path. Consequently, the elastica has become a case study for bifurcation theory and persists as a focal point for analytical mechanics.

There are several standard means of depicting the results from solutions to the elastica graphically. One is $x-y$ plots of the shapes of the elastica in dimensionless coordinates $x$ and $y$ [4]. Another is various forcedisplacement plots $[4,5]$. Yet another is force-deflection plots $[4,6]$. Where solutions of elastica are discussed in the context of stability of their equilibrium configurations, bifurcation diagrams are very useful for depicting results [7]. In this paper, we depict results with $x-y$ plots and force-displacement plots which are relatively easy to obtain by numerical methods. However, in general, it is not possible to express the force as a function of displacement or $y$ as a function of $x$ explicitly.

The focus of this paper is the classical case of an intrinsically straight inextensible rod that is clamped at one end and pinned at the other end. A typical analytical treatment of this problem involves finding equilibrium

P. Singh $(\varangle)$. V. G. A. Goss

School of Engineering, London South Bank University, London SE1 OAA, UK

E-mail: singhp9@1sbu.ac.uk 
solutions as the pinned end is displaced by an amount $d$ towards, and eventually past, the clamped end, while keeping the ends aligned. That manner of loading the rod is referred to as rigid loading [8]. In this paper, $d$ is the dimensionless end displacement of the pinned end. The clamped-pinned case has received relatively little attention compared to similar elastica problems involving symmetric boundary conditions, for example clamped-clamped and pinned-pinned cases [9]. Mikata noted that and presented a solution in terms of elliptic functions and integrals for the first mode that is applicable to displacements $d$ up to, but not beyond, the clamped end [10]. Tongyun adopts a numerical approach based on constrained optimisation to solving the nonlinear ordinary differential equations (odes) of the clamped-pinned elastica for the first mode for displacements $d$ of the pinned end towards, and past, the clamped end. The results are displayed mainly in the form of $x-y$ plots and load-displacement plots [4]. Humer encompasses the clamped-pinned case in a study of the exact solutions of shear-deformable rods [6]. The results of that study, as well as providing exact solutions for equilibrium paths for any mode, include load-displacement plots for the first two modes and $x-y$ plots for the first three modes. Humer also identifies, as we will do, equilibrium paths for the $n$th mode with $n+1$ or $n$ inflection points. However, no correlation is made between the number of inflection points and the domain of displacement $d$. Neither is the vanishing of an inflection point discussed (which occurs for the value of $d$ corresponding to the two end points of the rod coinciding).

In addition to exhibiting solutions representing post-buckling equilibrium configurations, the forcedisplacement plots for the clamped-pinned elastica are characterised by the presence of local extrema. Wang draws attention to those extrema by associating them with jump phenomena [5]; see also Banu et al. [11]. The stability of the post-buckling equilibrium configurations of the inextensible elastic rod under various loading conditions has been analysed using different techniques. Levyakov and Kuznetzov formulate a functional for the strain energy of the rod in the presence of isoperimetric constraints [12]. The stability of a configuration is determined by the sign of the second variation of that functional; i.e. if the second variation is positive definite, the configuration is stable. By representing the second variation of that functional in terms of eigensolutions of an auxiliary Sturm-Liouville problem, Levyakov and Kuznetzov determine that a configuration is stable if all eigenvalues lie outside the unit interval $[0,1]$. However, they do not appear to attempt to locate the critical points at which changes in stability arise. A similar, if not the same, energy-based approach for testing for the stability of post-buckled equilibrium configurations of the elastica is found in Bigoni et al. [9]. Batista presents an alternative approach to determining the stability of post-buckled equilibrium configurations of inextensible elastic rods based on the Jacobi test. Again, a functional which represents the total potential energy of the deformed rod is constructed. This time the sign of the second variation is determined via the Jacobi test. That test is demonstrated successfully on a cantilever with a free and sliding end, but fails to work for the pinnedpinned case [13]. In a subsequent paper, Batista applies the Jacobi test to the clamped-clamped elastic rod in any mode and the clamped-pinned elastic rod in the first mode. For the latter, a force-displacement diagram is presented showing a region, between two critical points, for which the rod is unstable [14].

Clearly, the stability of elastica solutions is currently a key focus of research, and it is useful to have information on critical points to aid stability analysis. This paper aims to contribute to that body of knowledge. We present a procedure which determines critical points of the clamped-pinned elastica and suggest how our procedure has scope for wider application.

\section{The clamped-pinned elastica}

We consider an elastic rod parameterised by its dimensionless (normalised) arc length, $0 \leq s \leq 1$ in the $x y$-plane, where $x$ and $y$ are dimensionless coordinates. The end points of the rod are fixed along the $x$-axis by a clamp at $s=0$ and a pinned joint at $s=1$ which can be displaced along the $x$-axis by (dimensionless) amount $d$, where $-2 \leq d \leq 0$. The rod is inextensible and unshearable but can deform in the $x y$-plane, under rigid loading through the parameter $d$. The dimensionless forces acting along the $x$-axis and $y$-axis are, respectively, $t$ and $r$. The tangent at any point $(x(s), y(s))$ along the bent rod is given by $\psi(s)$; see Fig. 1 . The equations appertaining to the static configuration and equilibrium of the rod are

$$
\begin{aligned}
\frac{\mathrm{d}^{2} \psi}{\mathrm{d} s^{2}} & =t \sin (\psi)-r \cos (\psi), \\
\frac{\mathrm{d} r}{\mathrm{~d} s} & =0 \\
\frac{\mathrm{d} t}{\mathrm{~d} s} & =0
\end{aligned}
$$




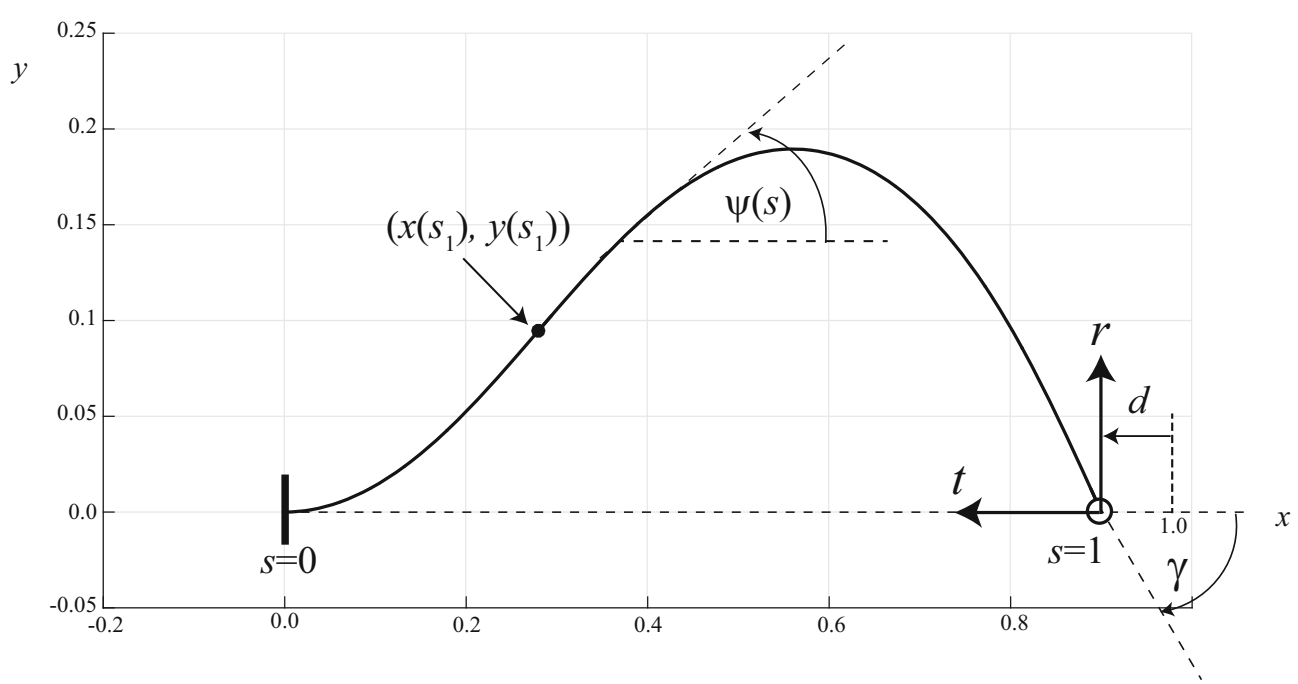

Fig. 1 Configuration of the rod for displacement of the pinned end along the $x$-axis by amount $d=x(1)-1=-0.1$. The rod is clamped at $s=0$ and pinned at $s=1$. For this value of $d$, the inflection point at $s_{1}=0.30$ has coordinates $\left(x\left(s_{1}\right), y\left(s_{1}\right)\right)=(0.28,0.09)$. For $0 \leq s<s_{1}$, the rod has buckled upwards into a 'sagging' form $(\smile)$ from the clamped end to the first inflection point which we denote with positive curvature. At the inflection point, the curvature changes to a 'hogging' form $(\frown)$ up to the end $s=1$, i.e. $s_{1} \leq s \leq 1$, which we denote as negative curvature

$$
\begin{aligned}
& \frac{\mathrm{d} x}{\mathrm{~d} s}=\cos (\psi), \\
& \frac{\mathrm{d} y}{\mathrm{~d} s}=\sin (\psi) .
\end{aligned}
$$

The boundary conditions are

$$
\begin{aligned}
x(0) & =0, \\
x(1) & =d+1, \\
y(0) & =0, \\
\psi(0) & =0, \\
\frac{\mathrm{d} \psi(1)}{\mathrm{d} s} & =0, \\
y(1) & =0 .
\end{aligned}
$$

We note that the value of the angle $\psi$ at the pinned end $(s=1)$ is designated $\gamma$, i.e. $\psi(1)=\gamma$.

\section{Solutions for post-buckling}

We introduce $f, \alpha$, and $\theta$ as

$$
f=\left(r^{2}+t^{2}\right)^{\frac{1}{2}}, \quad \alpha=\arctan \left(\frac{r}{-t}\right), \quad \theta=\psi+\alpha
$$

where $f$ is the dimensionless resultant force. Consequently, our system of Eqs. (1)-(5) becomes

$$
\begin{aligned}
\frac{\mathrm{d}^{2} \theta}{\mathrm{d} s^{2}} & =-f \sin (\theta), \\
\frac{\mathrm{d} f}{\mathrm{~d} s} & =0, \\
\frac{\mathrm{d} \alpha}{\mathrm{d} s} & =0,
\end{aligned}
$$




$$
\begin{aligned}
& \frac{\mathrm{d} x}{\mathrm{~d} s}=\cos (\alpha) \cos (\theta)+\sin (\alpha) \sin (\theta), \\
& \frac{\mathrm{d} y}{\mathrm{~d} s}=\cos (\alpha) \sin (\theta)-\sin (\alpha) \cos (\theta),
\end{aligned}
$$

and the boundary conditions Eqs. (6)-(11) become

$$
\begin{aligned}
x(0) & =0, \\
x(1) & =d+1, \\
y(0) & =0, \\
\theta(0) & =\alpha, \\
\frac{\mathrm{d} \theta(1)}{\mathrm{d} s} & =0, \\
y(1) & =0 .
\end{aligned}
$$

From Eqs. (17), (20) and (23), we find that

$$
\cos (\alpha) \int_{0}^{1} \sin (\theta(s)) \mathrm{d} s-\sin (\alpha) \int_{0}^{1} \cos (\theta(s)) \mathrm{d} s=0
$$

and from Eqs. (16), (18) and (19), we note that

$$
x(1)=\cos (\alpha) \int_{0}^{1} \cos (\theta(s)) \mathrm{d} s+\sin (\alpha) \int_{0}^{1} \sin (\theta(s)) \mathrm{d} s=d+1 .
$$

Integrating Eq. (13) and applying the boundary condition Eq. (22), we obtain, using the value at $\theta(1)=$ $\gamma+\alpha$

$$
\frac{\mathrm{d} \theta}{\mathrm{d} s}= \pm 2 f^{\frac{1}{2}}\left(\sin ^{2}\left(\frac{\gamma+\alpha}{2}\right)-\sin ^{2}\left(\frac{\theta}{2}\right)\right)^{\frac{1}{2}}= \pm 2 f^{\frac{1}{2}}\left(k^{2}-\sin ^{2}\left(\frac{\theta}{2}\right)\right)^{\frac{1}{2}}
$$

where $\frac{\mathrm{d} \theta}{\mathrm{d} s} \equiv \frac{\mathrm{d} \psi}{\mathrm{d} s}$ is the curvature. Anticipating elliptic integrals, we introduce the elliptic modulus

$$
k=\sin \left(\frac{(-1)^{n}(\gamma+\alpha)}{2}\right), \quad n=1,2,3, \ldots
$$

where $n$ is the mode number. Also the elliptic argument is

$$
\phi_{\theta}=\arcsin \left(\frac{1}{k} \sin \left(\frac{\theta}{2}\right)\right)
$$

and for the case $\theta=\alpha$ we have

$$
\phi_{\alpha}=\arcsin \left(\frac{1}{k} \sin \left(\frac{\alpha}{2}\right)\right)
$$

For each mode, the post-buckled configurations are inflectional. The $n$th mode has $n+1$ inflection points for $-1 \leq d \leq 0$ and $n$ inflection points for $-2 \leq d<-1$; i.e. as the pinned end moves past the clamped end, the inflection point closest to the clamped end vanishes, as discussed below. 


\subsection{Case $d \neq-1$}

The clamped-pinned rod buckles at critical loads $t=-t_{c}$, where $t_{c}$ is determined by the following transcendental equation:

$$
\tan \left(t_{c}^{\frac{1}{2}}\right)=t_{c}^{\frac{1}{2}}
$$

see Timoshenko and Gere [15]. The solutions of Eq. (30) are $t_{c}=20.19072856,59.67951594$, $118.89986916, \ldots$, and so on. The $n$th solution of Eq. (30) corresponds to the $n$th buckling mode and has $n+1$ inflection points.

The value of $s$ at the $i$ th inflection point is denoted by $s_{i}$. For $-1<d<0$, inflection points occur when

$$
\left.\frac{\mathrm{d} \theta}{\mathrm{d} s}\right|_{s_{i}}=0, \quad i=1,2,3, \ldots, n, n+1 .
$$

The curvature $\mathrm{d} \theta / \mathrm{d} s>0$ for $0 \leq s<s_{1}, \mathrm{~d} \theta / \mathrm{d} s<0$ for $s_{1} \leq s<s_{2}, \mathrm{~d} \theta / \mathrm{d} s>0$ for $s_{2} \leq s<s_{3}, \ldots$, and $\mathrm{d} \theta / \mathrm{d} s<0$ for $n$ odd and $\mathrm{d} \theta / \mathrm{d} s>0$ for $n$ even for $s_{n} \leq s \leq s_{n+1}=1$.

Applying Eq. (31) to Eq. (26), we find that inflection points arise when

$$
\sin \left(\frac{\theta_{i}}{2}\right)= \pm k= \pm \sin \left(\frac{(-1)^{n}(\gamma+\alpha)}{2}\right) \Longrightarrow \theta_{i}=(-1)^{(n+i-1)}(\gamma+\alpha) \quad i=1,2,3, \ldots, n, n+1
$$

Note that $\theta\left(s_{n+1}\right)=\theta(1)=\gamma+\alpha$ corresponds to the inflection point at the pinned end. Integration, from 0 to $s_{1}$ and between successive pairs of inflection points $s_{i-1}$ to $s_{i}$, for $i=2,3,4, \ldots, n, n+1$ of Eq. (26) gives

$$
1=\frac{1}{f^{\frac{1}{2}}}\left[(2 n+1) K(k)-F\left(\phi_{\alpha}, k\right)\right]
$$

where $K(k)$ and $F\left(\phi_{\alpha}, k\right)$ are respectively the complete and incomplete elliptic integrals of the first kind. From Eq. (24), using Eqs. (27), (29) and (33) we obtain

$$
2 \cos (\alpha)\left(k^{2}-\sin ^{2}\left(\frac{\alpha}{2}\right)\right)^{\frac{1}{2}}-\sin (\alpha)\left[(2 n+1)(2 E(k)-K(k))-2 E\left(\phi_{\alpha}, k\right)+F\left(\phi_{\alpha}, k\right)\right]=0
$$

where $E(k)$ and $E\left(\phi_{\alpha}, k\right)$ are, respectively, the complete and incomplete elliptic integrals of the second kind. Integration of Eq. (25), using Eq. (34), gives

$$
d+1=\frac{2}{f^{\frac{1}{2}} \sin (\alpha)}\left(k^{2}-\sin ^{2}\left(\frac{\alpha}{2}\right)\right)^{\frac{1}{2}} .
$$

When $-1<d<0$, the clamped-pinned elastica has $n+1$ inflection points in the $n$th mode. Therefore, when the rod buckled in the first mode, there are two inflection points at $s_{1}$ and $s_{2}$, where $0<s_{1}<1$ and $s_{2}=1$. In this configuration, $\frac{\mathrm{d} \theta(0)}{\mathrm{d} s}>0, \frac{\mathrm{d} \theta\left(s_{1}\right)}{\mathrm{d} s}=0$ and $\frac{\mathrm{d} \theta\left(s_{2}\right)}{\mathrm{d} s}=0$. Taking the limit $d \rightarrow-1^{+}$, we find using Eqs. (21), (26) and (35) that $\frac{\mathrm{d} \theta(0)}{\mathrm{d} s} \rightarrow 0$. Thus, the inflection point at $s_{1}>0$ for $d>-1$ has moved to $s_{1}=0$ for $d=-1$. If the pinned end is pulled beyond $d<-1$, we get $\frac{\mathrm{d} \theta(0)}{\mathrm{d} s}<0$, and so the inflection point at $s_{1}=0$ for $d=-1$ has either vanished or has moved to some $s_{1}>0$ for $d<-1$. The forces arising in the rod as a consequence of decreasing $d$ beyond $d<-1$ are now pulling out the pinned end towards $d=-2$. Such forces will have a tendency to straighten out the rod and eliminate the inflection point rather than move it. Thus, the inflection point at $s_{1}$ must vanish; i.e. it is effectively pulled out of the rod (see Fig. 2). This is contrary to the situation when $-1<d \leq 0$ and the forces in the rod act to compress an inflection point into the rod at $s_{1}$. So, for $-2 \leq d<-1$ we have one less inflection points than we had for $-1 \leq d \leq 0$. Similar arguments can be applied to higher modes. Thus, for all modes, the inflection point at $s_{1}>0$ for $d>-1$ moves to $s_{1}=0$ for $d=-1$ and then vanishes when $d<-1$. Hence, if there are $n+1$ inflection points for $-1 \leq d<0$, then there are $n$ inflection points for $-2<d<-1$. 
As before, the value of $s$ at the $i$ th inflection point is denoted by $s_{i}$, and inflection points occur when

$$
\left.\frac{\mathrm{d} \theta}{\mathrm{d} s}\right|_{s_{i}}=0, \quad i=1,2,3, \ldots, n .
$$

For this case, the curvature $\mathrm{d} \theta / \mathrm{d} s<0$ for $0 \leq s<s_{1}, \mathrm{~d} \theta / \mathrm{d} s>0$ for $s_{1} \leq s<s_{2}, \mathrm{~d} \theta / \mathrm{d} s<0$ for $s_{2} \leq s<s_{3}, \ldots$, and $\mathrm{d} \theta / \mathrm{d} s<0$ for $n$ odd and $\mathrm{d} \theta / \mathrm{d} s>0$ for $n$ even for $s_{n-1} \leq s \leq s_{n}=1$. Applying Eq. (36) to Eq. (26), it follows that inflection points arise when

$$
\sin \left(\frac{\theta_{i}}{2}\right)= \pm k= \pm \sin \left(\frac{(-1)^{n}(\gamma+\alpha)}{2}\right) \Longrightarrow \theta_{i}=(-1)^{(n+i)}(\gamma+\alpha) \quad i=1,2,3, \ldots, n .
$$

Note that $\theta\left(s_{n}\right)=\theta(1)=\gamma+\alpha$ corresponds to the inflection point at the pinned end. Integration, from 0 to $s_{1}$ and between successive pairs of inflection points $s_{i-1}$ to $s_{i}$, for $i=2,3,4, \ldots, n$ of Eq. (26) gives

$$
1=\frac{1}{f^{\frac{1}{2}}}\left[(2 n-1) K(k)+F\left(\phi_{\alpha}, k\right)\right] .
$$

From Eq. (24), using Eqs. (27), (29) and (38), we obtain

$$
2 \cos (\alpha)\left(k^{2}-\sin ^{2}\left(\frac{\alpha}{2}\right)\right)^{\frac{1}{2}}+\sin (\alpha)\left[(2 n-1)(2 E(k)-K(k))+2 E\left(\phi_{\alpha}, k\right)-F\left(\phi_{\alpha}, k\right)\right]=0 .
$$

Integration of Eq. (25), using Eq. (39), gives

$$
d+1=-\frac{2}{f^{\frac{1}{2}} \sin (\alpha)}\left(k^{2}-\sin ^{2}\left(\frac{\alpha}{2}\right)\right)^{\frac{1}{2}} .
$$

At this point, we observe that the equations for $-1<d<0$ and $-2<d<-1$ differ by \pm signs and the highest value the index $i$ takes. Henceforth, for $d \neq-1$ denote this highest value of $i$ by $I$, where $I=n+1$ for $-1<d<0$ and $I=n$ for $-2<d<-1$. Furthermore, if \pm or $\mp$ signs appear in any subsequent equations for $d \neq-1$, let the upper sign refer to $-1<d<0$ and lower sign refer to $-2<d<-1$. Then, the following expressions for $s, x$, and $y$ can be derived from Eqs. (16), (17) and (26), using Eqs. (27)-(29), and are applicable for $s_{i-1} \leq s \leq s_{i}$, for $i=1,2,3, \ldots, I$, where $s_{0}=0, s_{I}=1$,

$$
\begin{aligned}
s= & \frac{1}{f^{\frac{1}{2}}}\left[2(i-1) K(k) \mp F\left(\phi_{\alpha}, k\right) \mp(-1)^{i} F\left(\phi_{\theta}, k\right)\right], \\
x= & \frac{\cos (\alpha)}{f^{\frac{1}{2}}}\left[4(i-1) E(k)-2(i-1) K(k) \mp 2 E\left(\phi_{\alpha}, k\right) \pm F\left(\phi_{\alpha}, k\right) \mp(-1)^{i} 2 E\left(\phi_{\theta}, k\right) \pm(-1)^{i} F\left(\phi_{\theta}, k\right)\right] \\
& \pm \frac{2 \sin (\alpha)}{f^{\frac{1}{2}}}\left[\left(k^{2}-\sin ^{2}\left(\frac{\alpha}{2}\right)\right)^{\frac{1}{2}}+(-1)^{i}\left(k^{2}-\sin ^{2}\left(\frac{\theta}{2}\right)\right)^{\frac{1}{2}}\right], \\
y= & -\frac{\sin (\alpha)}{f^{\frac{1}{2}}}\left[4(i-1) E(k)-2(i-1) K(k) \mp 2 E\left(\phi_{\alpha}, k\right) \pm F\left(\phi_{\alpha}, k\right) \mp(-1)^{i} 2 E\left(\phi_{\theta}, k\right) \pm(-1)^{i} F\left(\phi_{\theta}, k\right)\right] \\
& \pm \frac{2 \cos (\alpha)}{f^{\frac{1}{2}}}\left[\left(k^{2}-\sin ^{2}\left(\frac{\alpha}{2}\right)\right)^{\frac{1}{2}}+(-1)^{i}\left(k^{2}-\sin ^{2}\left(\frac{\theta}{2}\right)\right)^{\frac{1}{2}}\right] .
\end{aligned}
$$

3.2 Case $d=-1$

Taking the limits $d \rightarrow-1^{ \pm}$, both Eqs. (33)-(35) and (38)-(40) reduce to the following equations:

$$
\begin{gathered}
1=\frac{2 n}{f^{\frac{1}{2}}} K(k), \\
2 E(k)-K(k)=0,
\end{gathered}
$$




$$
k-\sin \left(\frac{\alpha}{2}\right)=0,
$$

which are valid at $d=-1$. Furthermore, by taking the limits $d \rightarrow-1^{ \pm}$in Eqs. (41)-(43), we obtain expressions for $s, x$, and $y$ at $d=-1$, for $\frac{i-1}{n} \leq s \leq \frac{i}{n}$, for $i=1,2,3, \ldots, n$, where $s_{0}=0, s_{n}=1$,

$$
\begin{aligned}
& s=\frac{1}{f^{\frac{1}{2}}}\left[(2 i-1) K(k)+(-1)^{i} F\left(\phi_{\theta}, k\right)\right] \\
& x=\frac{\cos (\alpha)(-1)^{i}}{f^{\frac{1}{2}}}\left[2 E\left(\phi_{\theta}, k\right)-F\left(\phi_{\theta}, k\right)\right]-\frac{2 \sin (\alpha)(-1)^{i}}{f^{\frac{1}{2}}}\left(k^{2}-\sin ^{2}\left(\frac{\theta}{2}\right)\right)^{\frac{1}{2}}, \\
& y=-\frac{\sin (\alpha)(-1)^{i}}{f^{\frac{1}{2}}}\left[2 E\left(\phi_{\theta}, k\right)-F\left(\phi_{\theta}, k\right)\right]-\frac{2 \cos (\alpha)(-1)^{i}}{f^{\frac{1}{2}}}\left(k^{2}-\sin ^{2}\left(\frac{\theta}{2}\right)\right)^{\frac{1}{2}} .
\end{aligned}
$$

\subsection{Procedure for solving for $\alpha, k$, and $f$}

We use Eqs. (33)-(35) for $-1<d<0$ and Eqs. (38)-(40) for $-2<d<-1$ to determine values for $\alpha$, $k$, and $f$. Once these are known, we use them in Eqs. (41)-(43) to plot the shape for $d \neq-1$. For the case $d=-1$, we solve Eqs. (44)-(46) for $\alpha, k$, and $f$ and use Eqs. (47)-(49) to plot the shapes. See Figs. 2, 3 and 4. Our plots in Figs. 2 and 3 agree with Humer's [6].

The values of $\alpha, k$, and $f$ are obtained by solving a system of three nonlinear equations simultaneously for a given value of the rigid loading parameter $d$. Here, we present the governing equations for $\alpha, k$, and $f$ in a form generally required for nonlinear solvers. Explicitly, for $d \neq-1$ we have

$$
\begin{array}{r}
U(\alpha, k)=2 \cos (\alpha)\left(k^{2}-\sin ^{2}\left(\frac{\alpha}{2}\right)\right)^{\frac{1}{2}} \\
\mp \sin (\alpha)\left((2 n \pm 1)(2 E(k)-K(k)) \mp 2 E\left(\phi_{\alpha}, k\right) \pm F\left(\phi_{\alpha}, k\right)\right)=0, \\
V(d, \alpha, k)=(d+1)\left((2 n \pm 1) K(k) \mp F\left(\phi_{\alpha}, k\right)\right) \\
\mp \frac{2}{\sin (\alpha)}\left(k^{2}-\sin ^{2}\left(\frac{\alpha}{2}\right)\right)^{\frac{1}{2}}=0, \\
W(\alpha, k, f)=f^{\frac{1}{2}}-(2 n \pm 1) K(k) \pm F\left(\phi_{\alpha}, k\right)=0,
\end{array}
$$

and for $d=-1$, we have

$$
\begin{gathered}
U(k)=2 E(k)-K(k)=0, \\
V(\alpha, k)=k-\sin \left(\frac{\alpha}{2}\right)=0, \\
W(k, f)=f^{\frac{1}{2}}-2 n K(k)=0 .
\end{gathered}
$$

For $d \neq-1$, the dependence on $f$ has been factored out of the system of three nonlinear equations into $W$. Now, both $U$ and $V$ are independent of $f$ and can be solved simultaneously for $\alpha$ and $k$. Once $\alpha$ and $k$ are known, $W$ can be used to determine $f$. Furthermore, note that $U$ does not explicitly depend on $d$, i.e. $U=U(\alpha, k)$. The situation for $d=-1$ is even simpler, all we need to do is solve $U$ for $k$. Once $k$ is known, we use $V$ and $W$ to determine $\alpha$ and $f$, respectively.

\section{Rates of change and critical points}

\subsection{Expressions for $\frac{\mathrm{d} \alpha}{\mathrm{d} d}$ and $\frac{\mathrm{d} k}{\mathrm{~d} d}$}

In the previous Section, we found exact solutions for the clamped-pinned rod under rigid loading through the parameter $d$ in terms of elliptic integrals. Finding these solutions relied on first finding values for the three variables $\alpha=\alpha(d), k=k(d)$, and $f=f(d)$ by numerically solving a system of three nonlinear equations simultaneously for a given value of $d$. Once $\alpha, k$, and $f$ are known, we have completely determined the 


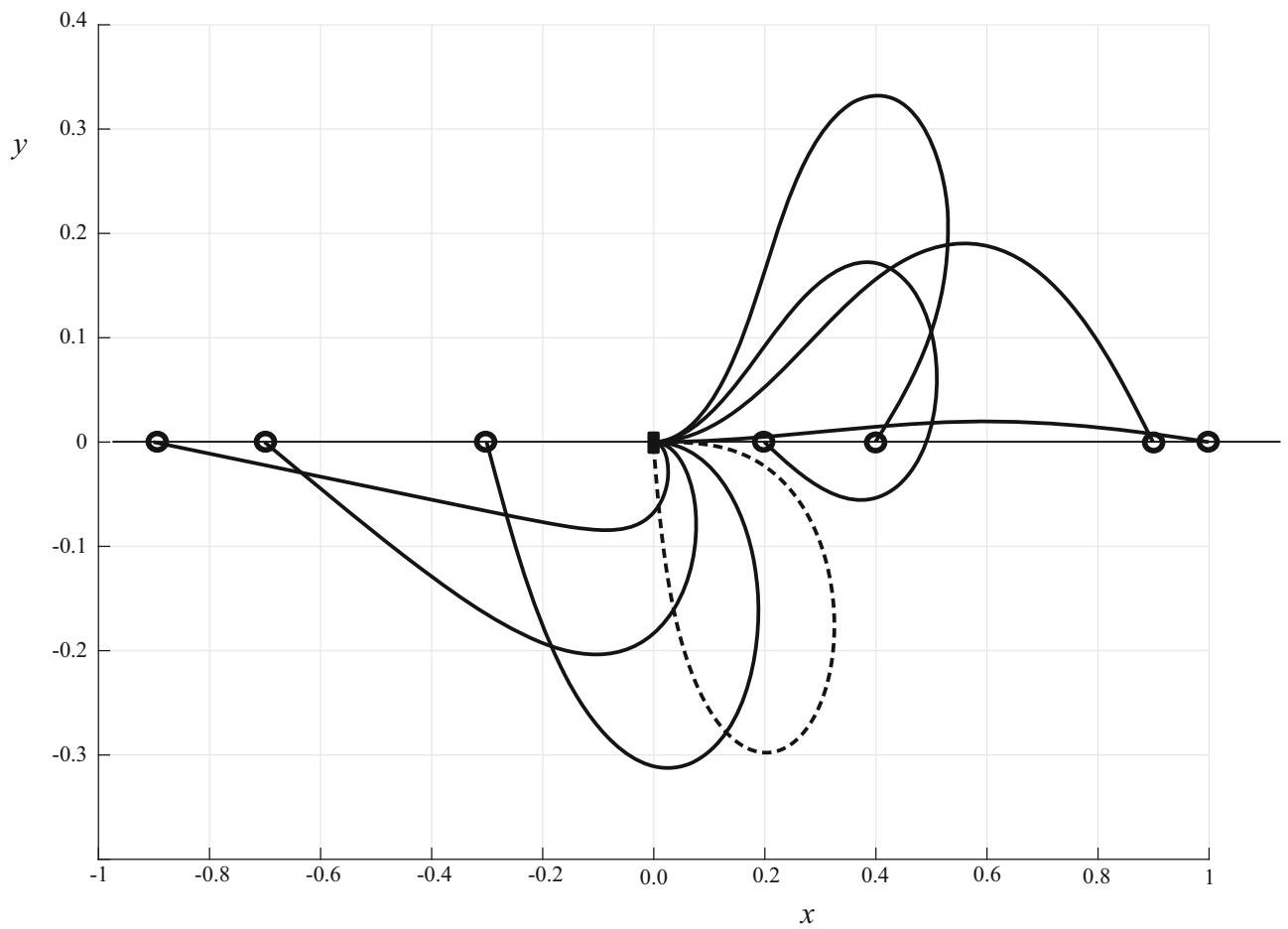

Fig. $2 x-y$ plots for various $d$ in the first mode. The dashed curve is the case $d=-1$
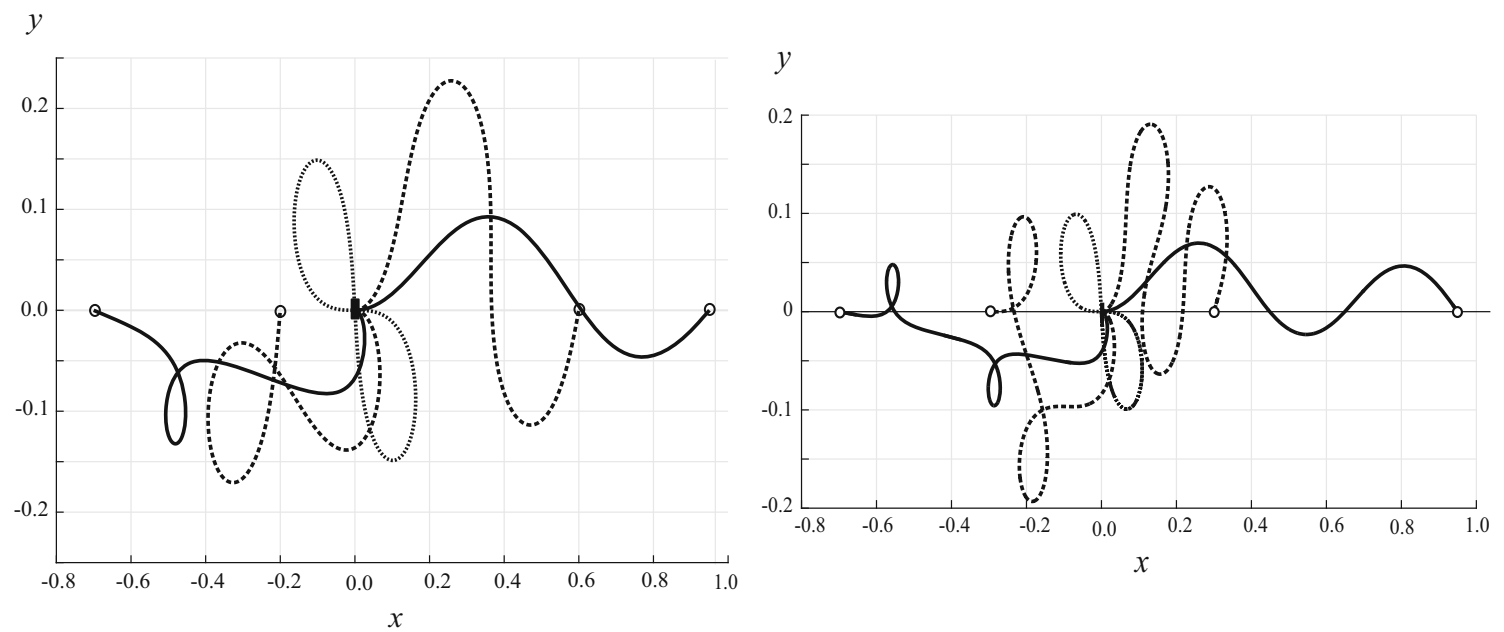

Fig. $3 x-y$ plots for various $d$ in the second and third modes, left and right, respectively

elastica as $d$ varies. However, we did not obtain closed-form analytic solutions for $\alpha=\alpha(d), k=k(d)$, and $f=f(d)$.

In practice, it is often useful to know rates of change of physical quantities and variables with respect to $d$ that describe the clamped-pinned rod. Those are useful for locating critical points and corresponding critical values. For example, in investigations on stability of solutions, we need to know where the critical points on $t d$ plots are. Therefore, we need to know $\frac{\mathrm{d} t}{\mathrm{~d} d}$. Not knowing the closed-form of $t=t(d)$ is not a hindrance for determining $\frac{\mathrm{d} t}{\mathrm{~d} d}$ as we will show. 

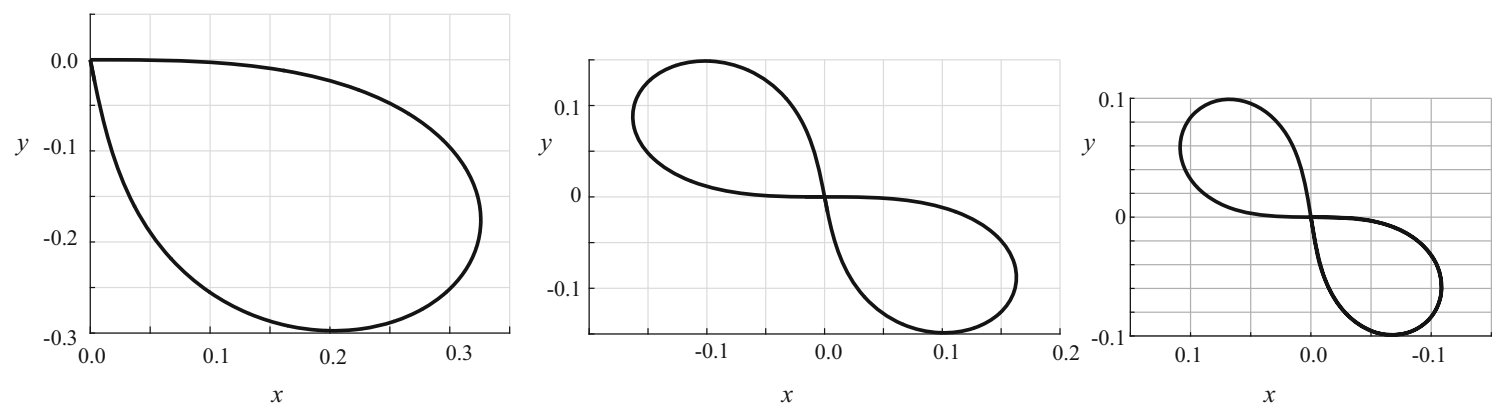

Fig. 4 Plots of the elastica at $d=-1$. In the first mode, a tear drop forms in the fourth quadrant (left). In the second mode, a second tear drop appears in the second quadrant forming a slanted 'figure of eight' (centre). In the third mode, a second tear drop overlaps the first. For each successive mode, another tear drop overlies successively those tear drops in the second and then fourth quadrants, etc, while the overall size of the loop diminishes maintaining unit length

We determine $\alpha, k$, and $f$ from Eqs. (50)-(55). For $d \neq-1$, we can show from Eqs. (50) and (51) that

$$
\mathbf{J}\left(\begin{array}{c}
\frac{\mathrm{d} \alpha}{\mathrm{d} d} \\
\frac{\mathrm{d} k}{\mathrm{~d} d}
\end{array}\right)=-\left(\begin{array}{c}
0 \\
\frac{\partial V}{\partial d}
\end{array}\right)
$$

where $\frac{\partial U}{\partial d}=0$ since $U=U(\alpha, k)$ and the $2 \times 2$ matrix $\mathbf{J}$ is given by

$$
\mathbf{J}=\left(\begin{array}{ll}
\frac{\partial U}{\partial \alpha} & \frac{\partial U}{\partial k} \\
\frac{\partial V}{\partial \alpha} & \frac{\partial V}{\partial k}
\end{array}\right) .
$$

Then, provided $\operatorname{det}(\mathbf{J}) \neq 0$ where

$$
\operatorname{det}(\mathbf{J})=\left(\frac{\partial U}{\partial \alpha} \frac{\partial V}{\partial k}-\frac{\partial U}{\partial k} \frac{\partial V}{\partial \alpha}\right)
$$

we find

$$
\begin{aligned}
& \frac{\mathrm{d} \alpha}{\mathrm{d} d}=\frac{\frac{\partial V}{\partial d} \frac{\partial U}{\partial k}}{\operatorname{det}(\mathbf{J})}, \\
& \frac{\mathrm{d} k}{\mathrm{~d} d}=-\frac{\frac{\partial V}{\partial d} \frac{\partial U}{\partial \alpha}}{\operatorname{det}(\mathbf{J})} .
\end{aligned}
$$

We now present all the partial derivatives of $U$ and $V$ that are required to evaluate quantities given by Eqs. (58)-(60). For $d \neq-1$,

$$
\begin{aligned}
& \frac{\partial U}{\partial \alpha}=-\frac{2}{\sin (\alpha)}\left(k^{2}-\sin ^{2}\left(\frac{\alpha}{2}\right)\right)^{\frac{1}{2}}=\mp(d+1) f^{\frac{1}{2}}, \\
& \frac{\partial U}{\partial k}=\frac{(d+2)}{(d+1)} \frac{\left(k^{2}-\sin ^{2}\left(\frac{\alpha}{2}\right)\right)^{\frac{1}{2}}}{k\left(1-k^{2}\right)}, \\
& \frac{\partial V}{\partial d}=f^{\frac{1}{2}}= \pm \frac{2}{(d+1)} \frac{\left(k^{2}-\sin ^{2}\left(\frac{\alpha}{2}\right)\right)^{\frac{1}{2}}}{\sin (\alpha)}, \\
& \frac{\partial V}{\partial \alpha}= \pm 2 \cos (\alpha) \frac{\left(k^{2}-\sin ^{2}\left(\frac{\alpha}{2}\right)\right)^{\frac{1}{2}}}{\sin ^{2}(\alpha)} \mp \frac{d}{2\left(k^{2}-\sin ^{2}\left(\frac{\alpha}{2}\right)\right)^{\frac{1}{2}}}, \\
& \frac{\partial V}{\partial k}= \pm \frac{(d+2)\left(k^{2}-\sin ^{2}\left(\frac{\alpha}{2}\right)\right)^{\frac{1}{2}}}{k\left(1-k^{2}\right) \sin ^{2}(\alpha)} \mp \frac{2\left(2 k^{2}-(d+2) \sin ^{2}\left(\frac{\alpha}{2}\right)\right)}{k \sin (\alpha)\left(k^{2}-\sin ^{2}\left(\frac{\alpha}{2}\right)\right)^{\frac{1}{2}}} .
\end{aligned}
$$


Now that all the partial derivatives of $U$ and $V$ are known, we can obtain equations for $\operatorname{det}(\mathbf{J})$. For $d \neq-1$,

$$
\begin{aligned}
\operatorname{det}(\mathbf{J})= & \mp \frac{2(d+2)\left(k^{2}-\sin ^{2}\left(\frac{\alpha}{2}\right)\right)}{k\left(1-k^{2}\right) \sin ^{2}(\alpha)} \pm \frac{4\left(2 k^{2}-(d+2) \sin ^{2}\left(\frac{\alpha}{2}\right)\right)}{k \sin ^{2}(\alpha)} \\
& \mp \frac{2(d+2) \cos (\alpha)\left(k^{2}-\sin ^{2}\left(\frac{\alpha}{2}\right)\right)}{(d+1) k\left(1-k^{2}\right) \sin ^{2}(\alpha)} \pm \frac{d(d+2)}{2(d+1) k\left(1-k^{2}\right)}
\end{aligned}
$$

On inspecting Eq. (66), we see that

- $\operatorname{det}(\mathbf{J}) \neq 0$ for $-2<d<0$.

- $\operatorname{det}(\mathbf{J}) \rightarrow-\infty$ as $d \rightarrow-1^{ \pm}$, i.e. $\operatorname{det}(\mathbf{J})$ is singular at $d=-1\left(\right.$ where $\left.k=\sin \left(\frac{\alpha}{2}\right)\right)$.

- $\operatorname{det}(\mathbf{J}) \rightarrow-\infty$ as $d \rightarrow-2^{+}$, i.e. $\operatorname{det}(\mathbf{J})$ is singular at $d=-2$ (where $\alpha \rightarrow 0, k \rightarrow 1$, and $f \rightarrow \infty$ $(t \rightarrow-\infty))$.

Finally, for $d \neq-1$

$$
\begin{aligned}
& \frac{\mathrm{d} \alpha}{\mathrm{d} d}= \pm \frac{\frac{2(d+2)\left(k^{2}-\sin ^{2}\left(\frac{\alpha}{2}\right)\right)}{(d+1)^{2} k\left(1-k^{2}\right) \sin (\alpha)}}{\operatorname{det}(\mathbf{J})} \\
& \frac{\mathrm{d} k}{\mathrm{~d} d}= \pm \frac{\frac{4\left(k^{2}-\sin ^{2}\left(\frac{\alpha}{2}\right)\right)}{(d+1) \sin ^{2}(\alpha)}}{\operatorname{det}(\mathbf{J})}
\end{aligned}
$$

Taking the limits $d \rightarrow-1^{ \pm}$in Eqs. (67) and (68) yields

$$
\begin{aligned}
& \frac{\mathrm{d} \alpha}{\mathrm{d} d}=0 \\
& \frac{\mathrm{d} k}{\mathrm{~d} d}=0
\end{aligned}
$$

at $d=-1$.

\subsection{Critical points on force-displacement plots}

\subsubsection{Resultant force-displacement $(f d)$ plot}

Equation (52) shows that for $d \neq-1, f$ has no explicit dependence on $d$, i.e. $f=f(\alpha, k)$, so $\frac{\partial f}{\partial d}=0$ and

$$
\frac{\mathrm{d} f}{\mathrm{~d} d}=\frac{\partial f}{\partial \alpha} \frac{\mathrm{d} \alpha}{\mathrm{d} d}+\frac{\partial f}{\partial k} \frac{\mathrm{d} k}{\mathrm{~d} d} .
$$

The partial derivatives of $f$, for $d \neq-1$ are

$$
\begin{aligned}
\frac{\partial f}{\partial \alpha}= & -\frac{2}{(d+1) \sin (\alpha)}, \\
\frac{\partial f}{\partial k}= & \frac{4\left(k^{2}-\sin ^{2}\left(\frac{\alpha}{2}\right)\right)}{(d+1) k \sin ^{2}(\alpha)}\left(\frac{1}{\left(1-k^{2}\right)}\left(\cos (\alpha)+\frac{1}{(d+1)}\right)-\frac{2}{(d+1)}\right) \\
& +\frac{2}{(d+1) k\left(1-k^{2}\right)} .
\end{aligned}
$$

Using Eqs. (67), (68), (72), and (73), we find that Eq. (71) gives for $d \neq-1$

$$
\frac{\mathrm{d} f}{\mathrm{~d} d}= \pm \frac{\left(\frac{4\left(k^{2}-\sin ^{2}\left(\frac{\alpha}{2}\right)\right)}{(d+1)^{2} k \sin ^{2}(\alpha)}\right)\left(\frac{d}{(d+1)\left(1-k^{2}\right)}+\frac{4\left(k^{2}-\sin ^{2}\left(\frac{\alpha}{2}\right)\right)}{\sin ^{2}(\alpha)}\left(\frac{1}{\left(1-k^{2}\right)}\left(\cos (\alpha)+\frac{1}{(d+1)}\right)-\frac{2}{(d+1)}\right)\right)}{\operatorname{det}(\mathbf{J})} .
$$


Table 1 Table of critical values $f_{\max }, f_{\min }$, and corresponding values of $d$

\begin{tabular}{lllll}
\hline Mode & $d$ & $f_{\min }$ & $d$ & $f_{\max }$ \\
\hline 1 & -1.34400169 & 13.29587535 & -0.78056639 & 30.63769034 \\
2 & -1.14999630 & 69.25505542 & -0.87970174 & 103.97412161 \\
3 & -1.09597864 & 168.31557539 & -0.91710908 & 220.40458719 \\
$\vdots$ & $\vdots$ & $\vdots$ & $\vdots$ & $\vdots$ \\
\hline
\end{tabular}

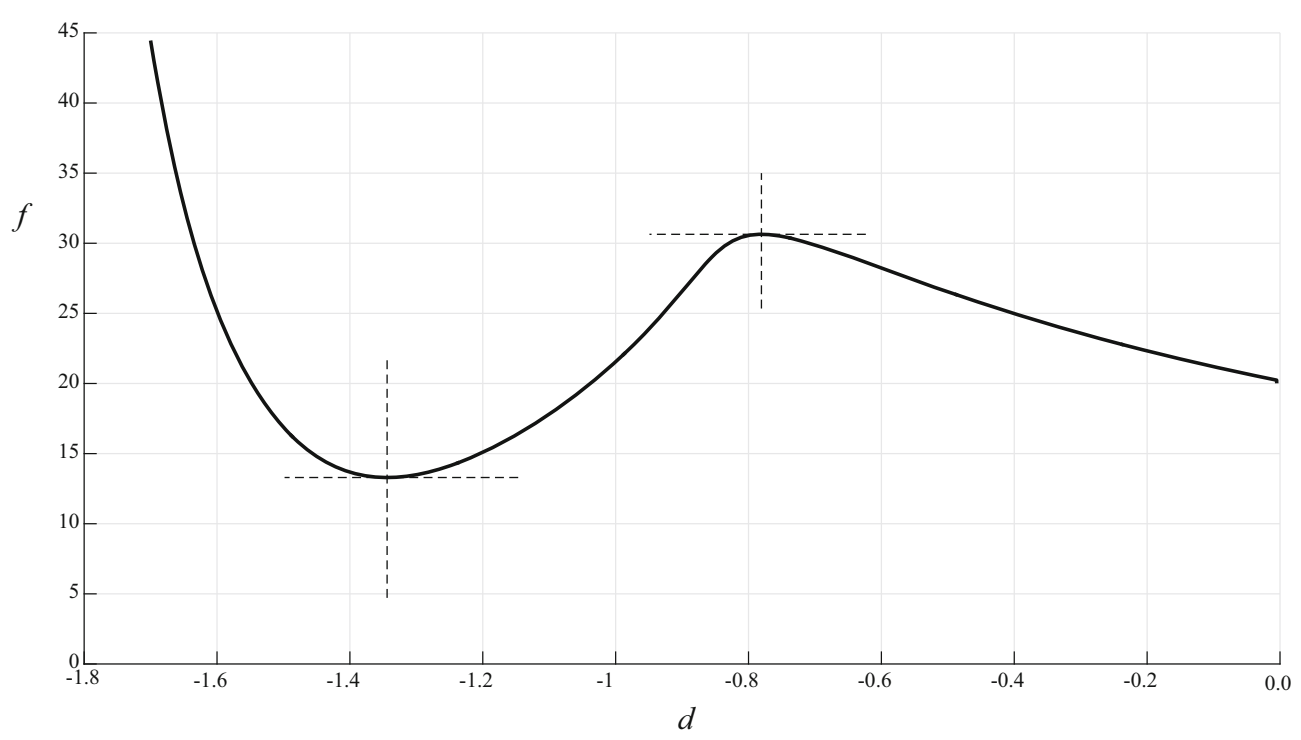

Fig. $5 f d$ plot for the first mode, showing the location of local maxima and minima, as given in Table 1

Taking the limits $d \rightarrow-1^{ \pm}$in Eq. (74), it can be shown that

$$
\frac{\mathrm{d} f}{\mathrm{~d} d}=2 f
$$

at $d=-1$. Thus, $\frac{\mathrm{d} f}{\mathrm{~d} d}$ is determined for $-2<d<0$.

At a critical point on $f, \frac{\mathrm{d} f}{\mathrm{~d} d}=0$. Since $f \neq 0$ anywhere in $-2<d<0$, Eq. (75) informs us that there is no critical point at $d=-1$. Thus, we need only consider $\frac{\mathrm{d} f}{\mathrm{~d} d}=0$ for $d \neq-1$ where the constraint equation for a critical point on $f$ from Eq. (74) is

$$
\frac{d}{(d+1)\left(1-k^{2}\right)}+\frac{4\left(k^{2}-\sin ^{2}\left(\frac{\alpha}{2}\right)\right)}{\sin ^{2}(\alpha)}\left(\frac{1}{\left(1-k^{2}\right)}\left(\cos (\alpha)+\frac{1}{(d+1)}\right)-\frac{2}{(d+1)}\right)=0 .
$$

Solving Eqs. (50) and (51) subject to (the constraint equation) Eq. (76), we obtain the locations $d$ (as well as $\alpha$ and $k$ ) of the critical points of $f$ tabulated in Table 1 . The corresponding critical values of $f$ are obtained from Eq. (52). Inspection of the $f d$ plots shown in Figs. 5 and 6 confirms the presence of critical points at the locations given in Table 1, predicted by our method.

\subsubsection{Horizontal force-displacement ( $t d$ ) plot}

Critical points on $t d$ plots are useful to know when considering the stability of solutions, especially for many physical rigid loading problems where it is the axial component of the force $t$ that is measured at the clamped end for a given input $d$; see, for example, [16]. From Eq. (12), we note that $t=t(\alpha, f)$, so

$$
\frac{\mathrm{d} t}{\mathrm{~d} d}=\frac{\partial t}{\partial \alpha} \frac{\mathrm{d} \alpha}{\mathrm{d} d}+\frac{\partial t}{\partial f} \frac{\mathrm{d} f}{\mathrm{~d} d}
$$



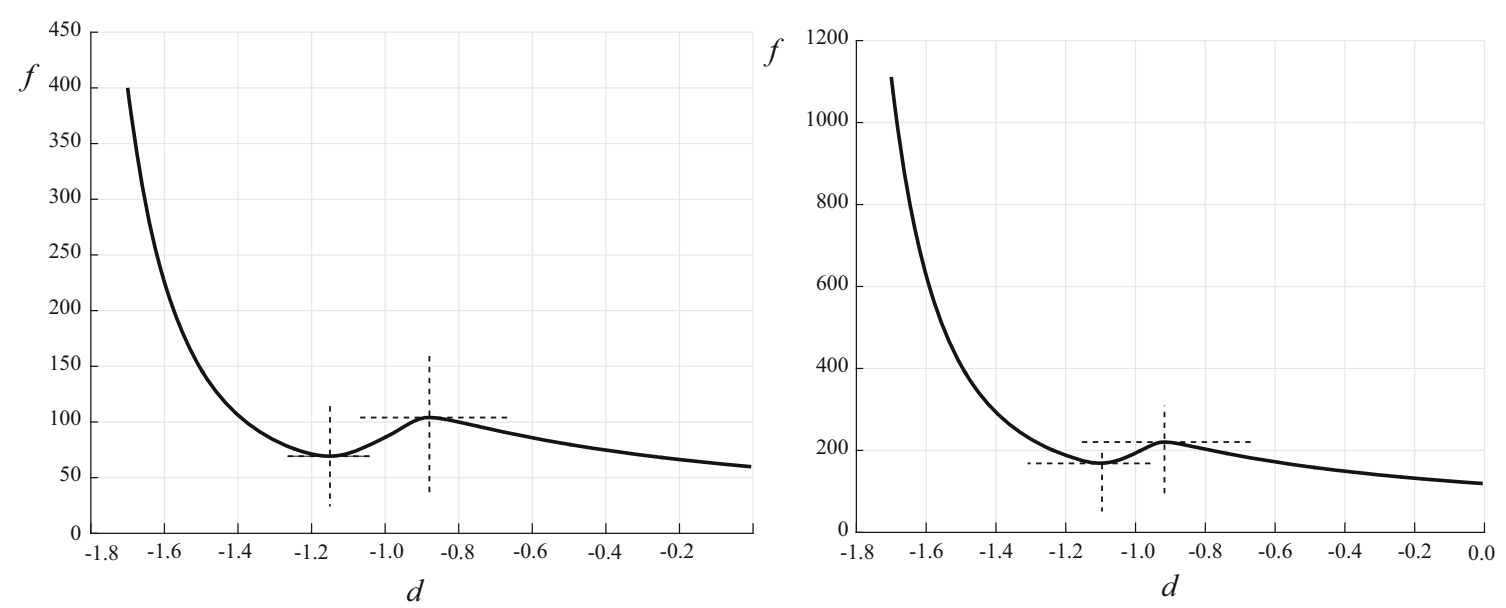

Fig. $6 \mathrm{fd}$ plots for the second mode (left) and third mode (right), showing the location of local maxima and minima, as given in Table 1

The partial derivatives of $t$, for $d \neq-1$ are

$$
\begin{aligned}
& \frac{\partial t}{\partial \alpha}=f \sin (\alpha)=\frac{4\left(k^{2}-\sin ^{2}\left(\frac{\alpha}{2}\right)\right)}{(d+1)^{2} \sin (\alpha)}, \\
& \frac{\partial t}{\partial f}=-\cos (\alpha) .
\end{aligned}
$$

Using Eqs. (67), (68), (78), and (79), we find that Eq. (77) gives for $d \neq-1$

$$
\frac{\mathrm{d} t}{\mathrm{~d} d}= \pm \frac{\left(\frac{4\left(k^{2}-\sin ^{2}\left(\frac{\alpha}{2}\right)\right)}{(d+1)^{3} k\left(1-k^{2}\right) \sin ^{2}(\alpha)}\right)\left(2\left(\frac{(d+2)}{(d+1)}+\frac{\left.2\left(1-2 k^{2}-(d+1) \cos (\alpha)\right) \cos (\alpha)\right)}{\sin ^{2}(\alpha)}\right)\left(k^{2}-\sin ^{2}\left(\frac{\alpha}{2}\right)\right)-d \cos (\alpha)\right)}{\operatorname{det}(\mathbf{J})}
$$

Taking the limits $d \rightarrow-1^{ \pm}$in Eq. (80), it can be shown that

$$
\frac{\mathrm{d} t}{\mathrm{~d} d}=2 t
$$

at $d=-1$. Thus, $\frac{\mathrm{d} t}{\mathrm{~d} d}$ is determined for $-2<d<0$.

At a critical point on $t, \frac{\mathrm{d} t}{\mathrm{~d} d}=0$. From the $t d$ plots, see Figs. 7 and 8 , it can be seen that $t \neq 0$ at $d=-1$, so Eq. (81) informs us that there is no critical point at $d=-1$. Thus, we need only consider $\frac{\mathrm{d} t}{\mathrm{~d} d}=0$ for $d \neq-1$ where the constraint equation for a critical point on $t$ from Eq. (80) is

$$
2\left(\frac{(d+2)}{(d+1)}+\frac{\left.2\left(1-2 k^{2}-(d+1) \cos (\alpha)\right) \cos (\alpha)\right)}{\sin ^{2}(\alpha)}\right)\left(k^{2}-\sin ^{2}\left(\frac{\alpha}{2}\right)\right)-d \cos (\alpha)=0 .
$$

Solving Eqs. (50) and (51) subject to (the constraint equation) Eq. (82), we obtain the locations $d$ (as well as $\alpha$ and $k$ ) of the critical points of $t$ set out in Table 2. The corresponding critical values of $t$ are obtained from Eqs. (12) and (52). Inspection of the $t d$ plots shown in Figs. 7 and 8 confirms the presence of critical points at the locations given in Table 2. Our results for the first mode agree with Humer [6], Levyakov and Kuznetsov [12], and Batista [14].

\subsubsection{Vertical force-displacement $(r d)$ plots}

Finally, consider the reaction force $r$ at the pinned end. Following reasoning identical to that in Sects. 4.2.1 and 4.2.2, the constraint equation for a critical point on $r$ is

$$
\frac{2\left(\left(2 d^{2}+5 d+4\right) \cos (\alpha)-2(d+1)\left(1-2 k^{2}\right)\right)}{(d+1) \sin ^{2}(\alpha)}\left(k^{2}-\sin ^{2}\left(\frac{\alpha}{2}\right)\right)+d=0
$$




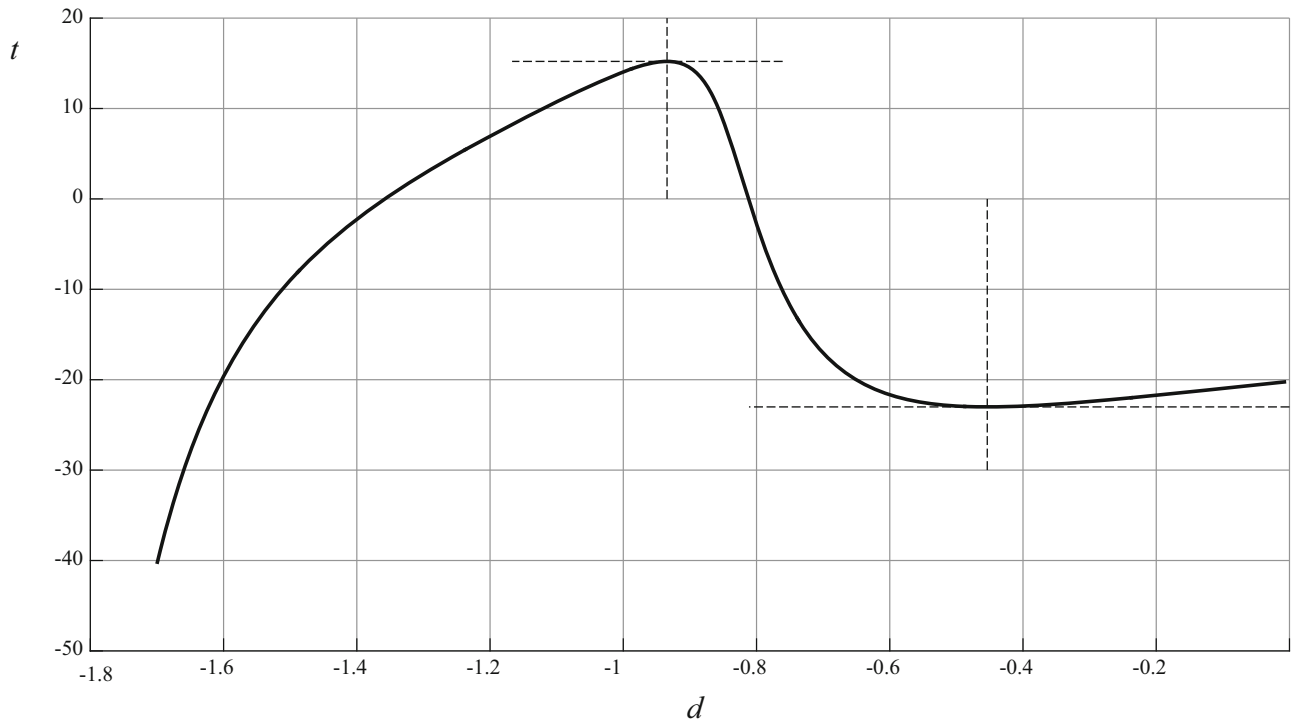

Fig. $7 \mathrm{td}$ plot for the first mode, showing the location of local maxima and minima, as given in Table 2
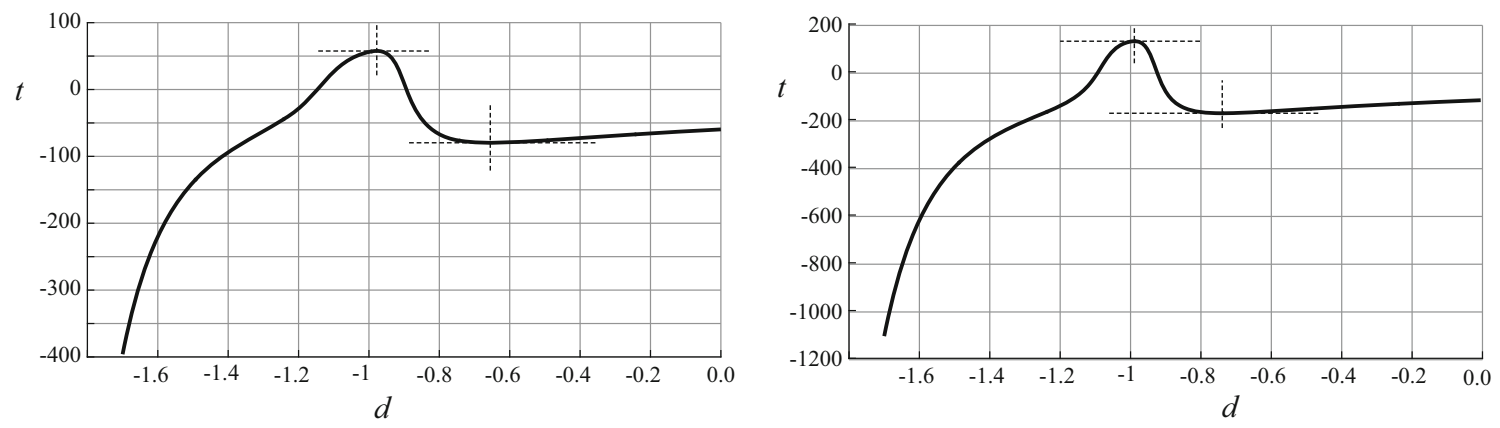

Fig. $8 t d$ plots for the second mode (left) and third mode (right), showing the location of local maxima and minima, as given in Table 2

Table 2 Table of critical values $t_{\min }, t_{\max }$, and corresponding values of $d$

\begin{tabular}{lllll}
\hline Mode & $d$ & $t_{\min }$ & $d$ & $t_{\max }$ \\
\hline 1 & -0.45364937 & -23.00958652 & -0.93432108 & 15.20954657 \\
2 & -0.65522709 & -79.66826933 & -0.97777821 & 57.57997021 \\
3 & -0.73945199 & -173.75751269 & -0.98923694 & 127.91560638 \\
$\vdots$ & $\vdots$ & $\vdots$ & $\vdots$ & $\vdots$ \\
\hline
\end{tabular}

where $d \neq-1$. Solving Eqs. (50) and (51) subject to (the constraint equation) Eq. (83), we obtain the locations $d$ (as well as $\alpha$ and $k$ ) of the critical points of $r$ displayed in Table 3. The corresponding critical values of $r$ are obtained from Eqs. (12) and (52). Inspection of the $r d$ plots shown in Figs. 9 and 10 confirms the presence of critical points at the locations given in Table 3. Our results for the first mode agree with Batista [14].

\subsection{Maximum deflection $h_{\max }$}

As the rod is compressed under the action of $d$, it bends into a hump, which arises between points of inflection. In the first mode, the $y$ deflection of the hump increases to a maximum height $h$ and then starts to decrease as the hump rotates round into the lower half-plane where another hump starts to form, the height of which attains another maximum (negative $y$ deflection) before it starts to get pulled out towards $x(1)=-1$ as 
Table 3 Table of critical values $r_{\min }, r_{\max }$, and corresponding values of $d$

\begin{tabular}{lllll}
\hline Mode & $d$ & $r_{\min }$ & $d$ & $r_{\max }$ \\
\hline 1 & -1.28752316 & 13.20564806 & -0.805201329 & 30.45974366 \\
2 & -1.04062397 & 62.87458767 & -0.891389242 & 103.73358440 \\
2 & & & -1.143492794 & 69.28089396 \\
3 & -1.10167120 & 144.62947128 & -0.924677967 & 220.10052006 \\
3 & $\vdots$ & $\vdots$ & $\vdots$ & -1.090368972 \\
$\vdots$
\end{tabular}

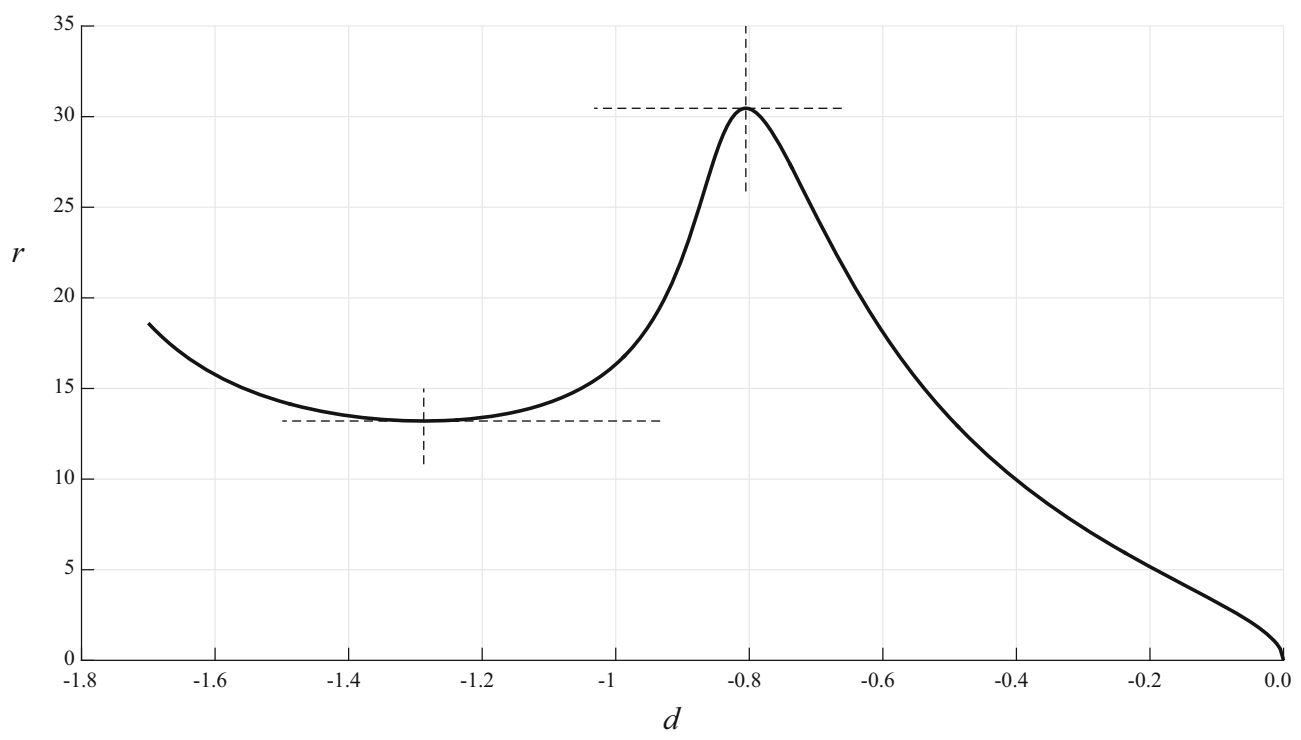

Fig. $9 r d$ plot for the first mode showing the location of local maxima and minima as given in Table 3
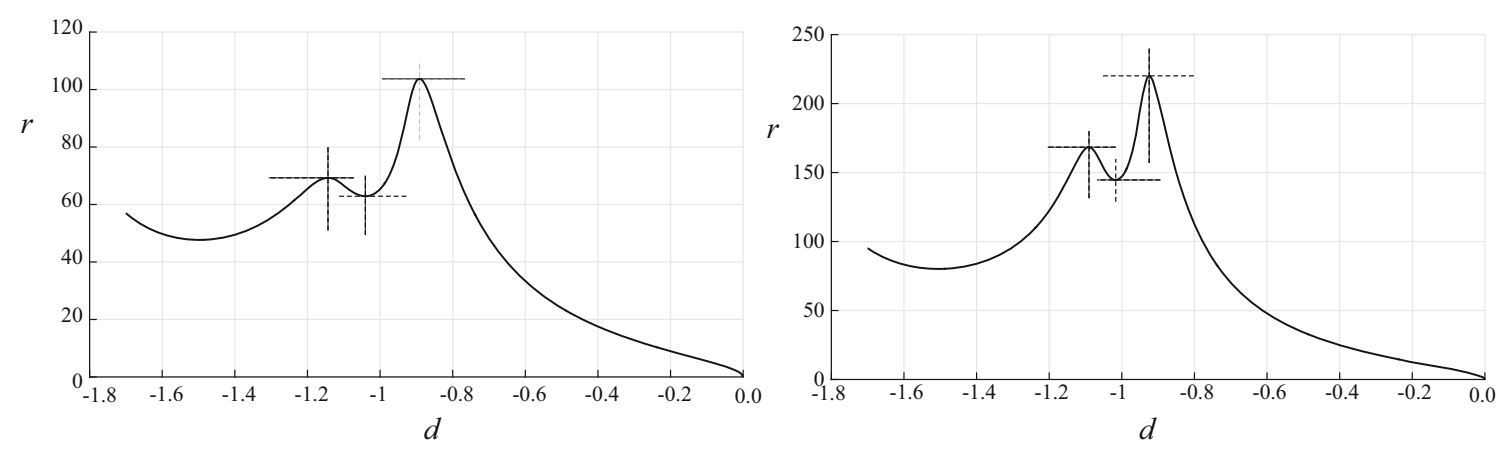

Fig. $10 r d$ plots for the second and third modes (left and right, respectively), showing the location of local maxima and minima, as given in Table 3

$d \rightarrow-2^{+}$; see Fig. 2. Inspection of plots of the shape of elastica indicates that the maximum height $h$ of the hump arises for values of end displacement $-1<d<0$. Furthermore, for the $n$th mode, there are $n$ humps for $-1<d<0$; however, the highest of those humps always emerges nearest the clamped end, i.e. between the first two inflection points $s_{1}$ and $s_{2}$, which is where we focus the analysis.

The relevant equation describing the behaviour of $y$ between the first two inflection points $s_{1}$ and $s_{2}$ when $-1<d<0$ is obtained from Eq. (43) with $i=2$ and is

$$
y=-\frac{\sin (\alpha)}{f^{\frac{1}{2}}}\left(4 E(k)-2 K(k)-2 E\left(\phi_{\alpha}, k\right)+F\left(\phi_{\alpha}, k\right)-2 E\left(\phi_{\theta}, k\right)+F\left(\phi_{\theta}, k\right)\right)
$$


Table 4 Table of maximum deflections $h_{\max }$ and corresponding values of $d$

\begin{tabular}{lll}
\hline Mode & $d$ & $h_{\max }$ \\
\hline 1 & -0.52261605 & 0.33907958 \\
2 & -0.61526626 & 0.24703476 \\
3 & -0.66227306 & 0.19131226 \\
$\vdots$ & $\vdots$ & $\vdots$ \\
\hline
\end{tabular}
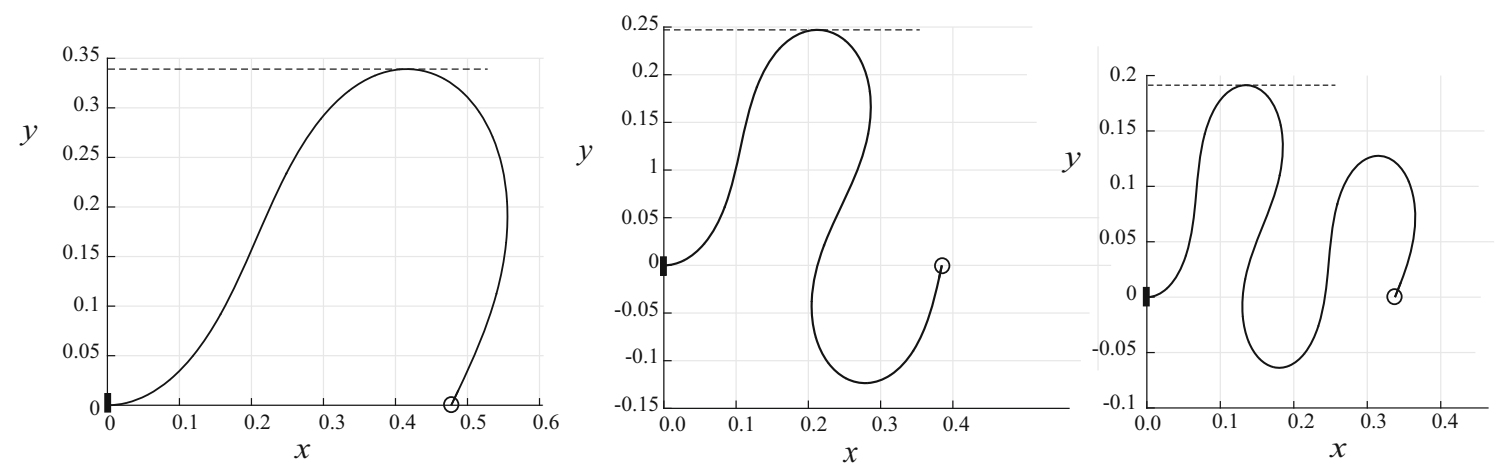

Fig. $11 x-y$ plots for the maximum deflection $h_{\max }$ for the first, second, and third modes, as given in Table 4

$$
+\frac{2 \cos (\alpha)}{f^{\frac{1}{2}}}\left(\left(k^{2}-\sin ^{2}\left(\frac{\alpha}{2}\right)\right)^{\frac{1}{2}}+\left(k^{2}-\sin ^{2}\left(\frac{\theta}{2}\right)\right)^{\frac{1}{2}}\right) .
$$

The maximum deflection for $y$, i.e. the height $h$ of the hump, occurs at $\theta=\alpha$. Setting $\theta=\alpha$ in Eq. (84) and using Eq. (34) gives

$$
h=\frac{4 n \sin (\alpha)}{f^{\frac{1}{2}}}(2 E(k)-K(k)) .
$$

To determine the maximum height $h_{\max }$ and the associated value of $d$ at which this occurs in $-1<d<0$ for the $n$th mode, we need to determine the condition obtained from $\frac{\mathrm{d} h}{\mathrm{~d} d}=0$. Since $h=h(\alpha, k, f)$, we have

$$
\frac{\mathrm{d} h}{\mathrm{~d} d}=\frac{\partial h}{\partial \alpha} \frac{\mathrm{d} \alpha}{\mathrm{d} d}+\frac{\partial h}{\partial k} \frac{\mathrm{d} k}{\mathrm{~d} d}+\frac{\partial h}{\partial f} \frac{\mathrm{d} f}{\mathrm{~d} d},
$$

where the partial derivatives of $h$ are given by

$$
\begin{aligned}
& \frac{\partial h}{\partial \alpha}=\frac{2 n(d+1)(2 E(k)-K(k)) \cos (\alpha) \sin (\alpha)}{\left(k^{2}-\sin ^{2}\left(\frac{\alpha}{2}\right)\right)^{\frac{1}{2}}}, \\
& \frac{\partial h}{\partial k}=\frac{2 n(d+1)\left(2 E(k)-K(k)-\frac{E(k)}{\left(1-k^{2}\right)}\right) \sin ^{2}(\alpha)}{k\left(k^{2}-\sin ^{2}\left(\frac{\alpha}{2}\right)\right)^{\frac{1}{2}}}, \\
& \frac{\partial h}{\partial f}=-\frac{n(d+1)^{3}(2 E(k)-K(k)) \sin ^{4}(\alpha)}{4\left(k^{2}-\sin ^{2}\left(\frac{\alpha}{2}\right)\right)^{\frac{3}{2}}} .
\end{aligned}
$$

Using Eqs. (67), (68), (74), (87)-(89), Eq. (86) gives an equation for $\frac{\mathrm{d} h}{\mathrm{~d} d}$. Setting this equation to zero gives the following constraint equation for $h$ to be a maximum:

$$
\begin{aligned}
& (2 E(k)-K(k))\left(\frac{d(d+1) \sin ^{2}(\alpha)}{2\left(k^{2}-\sin ^{2}\left(\frac{\alpha}{2}\right)\right)}+2\left(d^{2}+d-1\right) \cos (\alpha)+2(d+1)\left(4 k^{2}-3\right)\right) \\
& +4(d+1) E(k)=0 .
\end{aligned}
$$


Solving Eqs. (50) and (51) subject to (the constraint equation) Eq. (90), we obtain the value of $d$ (as well as $\alpha$ and $k$ ) at which the maximum height $h_{\max }$ occurs. The corresponding value of $f$ is obtained from Eq. (52). Those values of $d, \alpha, k$, and $f$ are substituted into Eq. (85) to give $h_{\max }$. Results for the first three modes are displayed in Table 4 . For the first mode, $h_{\max }$ rises to approximately one-third of the rod's length when the magnitude of the displacement $d$ exceeds half the rod's length; see Fig. 11. A similar result is presented by Tongyun [4].

\section{Conclusions}

Once $\alpha, k$, and $f$ are known, the clamped-pinned elastica under rigid loading parameterised by $d$ is completely determined, as shown above. We observed that $\alpha=\alpha(d), k=k(d)$, and $f=f(d)$; however, closed-form analytic solutions for $\alpha=\alpha(d), k=k(d)$ and $f=f(d)$ could not be found due to the nonlinearity in the governing equations for $\alpha, k$, and $f$, i.e. Eqs. (50)-(55). In spite of this, we found expressions for $\frac{\mathrm{d} \alpha}{\mathrm{d} d}$, $\frac{\mathrm{d} k}{\mathrm{~d} d}$, and $\frac{\mathrm{d} f}{\mathrm{~d} d}$ (see Eqs. (67)-(70) and (74), (75)). Then, given a physical quantity represented by $q=q(d, \alpha, k, f)$ in our clamped-pinned rod system above, we can determine its rate of change with respect to $d$ from

$$
\frac{\mathrm{d} q}{\mathrm{~d} d}=\frac{\partial q}{\partial d}+\frac{\partial q}{\partial \alpha} \frac{\mathrm{d} \alpha}{\mathrm{d} d}+\frac{\partial q}{\partial k} \frac{\mathrm{d} k}{\mathrm{~d} d}+\frac{\partial q}{\partial f} \frac{\mathrm{d} f}{\mathrm{~d} d} .
$$

This was precisely the approach we used, for example, for finding $\frac{\mathrm{d} t}{\mathrm{~d} d}$ given by Eqs. (80), (81). Once $\frac{\mathrm{d} q}{\mathrm{~d} d}$ is known, we can obtain the constraint equation for the presence of a critical point on $q$ by setting

$$
\frac{\mathrm{d} q}{\mathrm{~d} d}=0
$$

The constraint equation obtained from Eq. (92) is of the form $Q=Q(d, \alpha, k)$ (see Eqs. (76), (82), (83) and (90)) and is expressed as

$$
Q=0
$$

Again, this was precisely the approach we used for finding constraint equations for critical points on $f, t, r$, and $h$ given by Eqs. (76), (82), (83), and (90), respectively. Those constraint equations were solved in conjunction with the governing equations for $\alpha, k$, and $f$, i.e. Eqs. (50)-(55). As mentioned above, various plots such as Figs. 5, 6, 7, 8, 9, 10, and 11 confirm the presence of critical points at locations and with values predicted by our method. Those plots can be obtained from either solving the boundary value problem, Eqs. (13)-(17) together with Eqs. (18)-(23), or by solving the system of three nonlinear equations (50)-(55).

Motivated by this analysis, consider a generalisation of the above nonlinear system to one described by $n$ variables $x_{1}(u), x_{2}(u), x_{3}(u), \ldots, x_{n}(u)$ parameterised by $u$ with $n$ governing nonlinear equations of the form

$$
\begin{aligned}
U_{1}\left(u, x_{1}, x_{2}, x_{3}, \ldots, x_{n}\right) & =0, \\
U_{2}\left(u, x_{1}, x_{2}, x_{3}, \ldots, x_{n}\right) & =0, \\
U_{3}\left(u, x_{1}, x_{2}, x_{3}, \ldots, x_{n}\right) & =0, \\
& \vdots \\
U_{n}\left(u, x_{1}, x_{2}, x_{3}, \ldots, x_{n}\right) & =0 .
\end{aligned}
$$

Provided this nonlinear system has a solution for all $u$ belonging to some relevant domain, we suggest our approach above may be used to find $\frac{\mathrm{d} x_{1}}{\mathrm{~d} u}, \frac{\mathrm{d} x_{2}}{\mathrm{~d} u}, \frac{\mathrm{d} x_{3}}{\mathrm{~d} u}, \ldots, \frac{\mathrm{d} x_{n}}{\mathrm{~d} u}$ from

$$
\mathbf{J}\left(\begin{array}{c}
\frac{\mathrm{d} x_{1}}{\mathrm{~d} u} \\
\frac{\mathrm{d} x_{2}}{\mathrm{~d} u} \\
\frac{\mathrm{d} x_{3}}{\mathrm{~d} u} \\
\vdots \\
\frac{\mathrm{d} x_{n}}{\mathrm{~d} u}
\end{array}\right)=-\left(\begin{array}{c}
\frac{\partial U_{1}}{\partial u} \\
\frac{\partial U_{2}}{\partial u} \\
\frac{\partial U_{3}}{\partial u} \\
\vdots \\
\frac{\partial U_{n}}{\partial u}
\end{array}\right)
$$


provided $\operatorname{det}(\mathbf{J}) \neq 0$ where the $n \times n$ matrix $\mathbf{J}$ is given by

$$
\mathbf{J}=\left(\begin{array}{ccccc}
\frac{\partial U_{1}}{\partial x_{1}} & \frac{\partial U_{1}}{\partial x_{2}} & \frac{\partial U_{1}}{\partial x_{3}} & \ldots & \frac{\partial U_{1}}{\partial x_{n}} \\
\frac{\partial U_{2}}{\partial x_{1}} & \frac{\partial U_{2}}{\partial x_{2}} & \frac{\partial U_{2}}{\partial x_{3}} & \ldots & \frac{\partial U_{2}}{\partial x_{n}} \\
\frac{\partial U_{3}}{\partial x_{1}} & \frac{\partial U_{3}}{\partial x_{2}} & \frac{\partial U_{3}}{\partial x_{3}} & \ldots & \frac{\partial U_{3}}{\partial x_{n}} \\
\vdots & \vdots & \vdots & \ddots & \vdots \\
\frac{\partial U_{n}}{\partial x_{1}} & \frac{\partial U_{n}}{\partial x_{2}} & \frac{\partial U_{n}}{\partial x_{3}} & \ldots & \frac{\partial U_{n}}{\partial x_{n}}
\end{array}\right) .
$$

Then, a physical quantity in the nonlinear system described by Eqs. (94)-(97) can be represented by $q=$ $q\left(u, x_{1}, x_{2}, x_{3}, \ldots, x_{n}\right)$ that will have a rate of change with respect to $u$ given by

$$
\frac{\mathrm{d} q}{\mathrm{~d} u}=\frac{\partial q}{\partial u}+\frac{\partial q}{\partial x_{1}} \frac{\mathrm{d} x_{1}}{\mathrm{~d} u}+\frac{\partial q}{\partial x_{2}} \frac{\mathrm{d} x_{2}}{\mathrm{~d} u}+\frac{\partial q}{\partial x_{3}} \frac{\mathrm{d} x_{3}}{\mathrm{~d} u}+\cdots+\frac{\partial q}{\partial x_{n}} \frac{\mathrm{d} x_{n}}{\mathrm{~d} u} .
$$

Once $\frac{\mathrm{d} q}{\mathrm{~d} u}$ is known, the constraint equation for the presence of a critical point on $q$ is determined by setting

$$
\frac{\mathrm{d} q}{\mathrm{~d} u}=0
$$

and obtaining the aforementioned constraint equation $Q=Q\left(u, x_{1}, x_{2}, x_{3}, \ldots, x_{n}\right)$ in the form

$$
Q=0 \text {. }
$$

The critical points can then be found by solving Eqs. (94)-(97) subject to (the constraint equation) Eq. (102).

As the main focus of our work has been on determining constraint equations for locating critical points for physical quantities, such as $t$, in the clamped-pinned rod, we did not investigate $d=0$ and $d=-2$. Our main reason is that there are no critical points at these values of $d$. A thorough investigation of the solutions for the clamped-pinned rod as $d \rightarrow 0^{-}$and $d \rightarrow-2^{+}$is a prospect for future research in the context of asymptotic behaviour. Incidentally, Wang [5] has investigated (the equivalent of) $d \rightarrow 0^{-}$and $d \rightarrow-2^{+}$for the clamped-pinned rod in the first mode.

Acknowledgements This research was funded by London South Bank University. We would like to thank the reviewers for valuable comments and suggestions.

Open Access This article is distributed under the terms of the Creative Commons Attribution 4.0 International License (http:// creativecommons.org/licenses/by/4.0/), which permits unrestricted use, distribution, and reproduction in any medium, provided you give appropriate credit to the original author(s) and the source, provide a link to the Creative Commons license, and indicate if changes were made.

\section{References}

1. Euler, L.: Methodus inveniendi lineas curvas maximi minimivi proprietate gaudentes. Appendix 1: De curvis elasticis, Bousquet, Lausanne, and Geneva (1744)

2. Levien, R.: The elastica: a mathematical history. Technical report UCB/EECS-2008-103, EECS Department, University of California, Berkeley, August 2008. http://www2.eecs.berkeley.edu/Pubs/TechRpts/2008/EECS-2008-103.html

3. Goss, V.G.A.: The history of the planar elastica: insights into mechanics and scientific method. Sci. Educ. 18(8), 1057-1082 (2009). https://doi.org/10.1007/s11191-008-9166-2

4. Tongyun, W.: A numerical study of elastica using constrained optimisation method, diploma thesis. University of Singapore, Department of Civil Engineering (2004)

5. Wang, C.: Post-buckling of a clamped-simply supported elastica. Int. J. Non Linear Mech. 32(6), 1115-1122 (1997). https:// doi.org/10.1016/S0020-7462(96)00125-4. http://www.sciencedirect.com/science/article/pii/S0020746296001254

6. Humer, A.: Exact solutions for the buckling and postbuckling of shear-deformable beams. Acta Mech. 224(7), 1493-1525 (2013). https://doi.org/10.1007/s00707-013-0818-1

7. Maddocks, J.H.: Stability of nonlinearly elastic rods. Arch. Ration. Mech. Anal. 85(4), 311-354 (1984). https://doi.org/10. 1007/BF00275737

8. Thompson, J.M.T.: Stability of elastic structures and their loading devices. J. Mech. Eng. Sci. 3(2), 153-162 (1961)

9. Bigoni, D., Bosi, F., Misseroni, D., Dal Corso, F., Noselli, G.: New phenomena in nonlinear elastic structures: from tensile buckling to configurational forces. In: Bigoni, D. (ed.) Extremely Deformable Structures, pp. 55-135. Springer, Vienna (2015)

10. Mikata, Y.: Complete solution of elastica for a clamped-hinged beam, and its applications to a carbon nanotube. Acta Mech. 190(1), 133-150 (2007). https://doi.org/10.1007/s00707-006-0402-z 
11. Banu, S., Saha, G., Saha, S.: Multisegment integration technique for post-buckling analysis of a pinned-fixed slender elastic rod. BRAC Univ. J. 5(2), 1-7 (2008)

12. Kuznetsov, V., Levyakov, S.: Complete solution of the stability problem for elastica of Euler's column. Int. J. Non Linear Mech. 37(6), 1003-1009 (2002). https://doi.org/10.1016/S0020-7462(00)00114-1. http://www.sciencedirect.com/science/ article/pii/S0020746200001141

13. Batista, M.: A simplified method to investigate the stability of cantilever rod equilibrium forms. Mech. Res. Commun. 67, 13-17 (2015). https://doi.org/10.1016/j.mechrescom.2015.04.009. http://www.sciencedirect.com/science/article/ pii/S0093641315000877

14. Batista, M.: On stability of elastic rod planar equilibrium configurations. Int. J. Solids Struct. 72, 144-152 (2015). https:// doi.org/10.1016/j.ijsolstr.2015.07.024. http://www.sciencedirect.com/science/article/pii/S0020768315003303

15. Timoshenko, S., Gere, J.M.: Theory of Elastic Stability, 2nd edn. McGraw-Hill Book Co, New York (1961)

16. Goss, V.G.A., van der Heijden, G.H.M., Thompson, J.M.T., Neukirch, S.: Experiments on snap buckling, hysteresis and loop formation in twisted rods. Exp. Mech. 45(2), 101-111 (2005). https://doi.org/10.1007/BF02428182

Publisher's Note Springer Nature remains neutral with regard to jurisdictional claims in published maps and institutional affiliations. 